\title{
Ophthalmia Neonatorum
}

National Cancer Institute

\section{Source}

National Cancer Institute. Ophthalmia Neonatorum. NCI Thesaurus. Code C116815.

Inflammation of the conjunctiva in a newborn due to chemical or infectious causes.

Aseptic conjunctivitis is often related to the use of prophylactic medications for infectious conjunctivitis. Septic conjunctivitis is related to perinatal exposure to microorganisms. 